BIO Web of Conferences 1, 00087 (2011)

DOI: $10.1051 /$ bioconf $/ 20110100087$

(C) Owned by the authors, published by EDP Sciences, 2011

\title{
Training Stiffness perception: Knowledge of results and modality effects
}

\author{
Kinneret Teodorescu* Daniel Gopher ${ }^{*}$ Maria Korman ${ }^{*}$ \\ *Technion, Haifa, Israel \\ E-mail: kinneret_w@yahoo.com
}

\begin{abstract}
Perception of compliant objects demands integration of haptic and visual position information with force information. Multisensory interactions are ubiquitous in perception, even at early processing stages, and thus can potentially play a role in learning. In this study we explored humans' improvement on uni-sensory stiffness discrimination as a function of different sensory conditions and immediate knowledge of results (KR) during training. Two by two design was used: subjects were trained over two days on stiffness discrimination task with either matched visual-tactile, or tactile only stimuli and either with or without immediate feedback on their performance during training trials. Training resulted in both immediate but also latent, overnight learning in the proportion of correctly discriminated pairs of targets $(P C)$, in all groups. Discrimination decision time (DT) gains were obtained only during practice, while between sessions partial deterioration was evident. Affordance of visual information during training blocks resulted in higher PC during training blocks, but lower PC in the haptic-only retests. This finding challenges the notion that long-term unisensory learning mechanisms operate optimally under multisensory training conditions, at least for the combination of the visual and haptic modalities. We didn't find evidence that information feedback during training enhances discrimination ability in terms of $P C$. However, we found transient within-session effects of KR and visual-haptic trainings on DT: while visualhaptic training resulted in slower responses, $K R$ training induced faster responses.
\end{abstract}

\section{Introduction}

Stiffness sensitivity is crucial for perception and discrimination of objects and is essential for many complex tasks (Lederman \& Klatzky, 2009), including medical procedures such as surgery and teleoperation (Howell et al., 2008; Sherman, Cavusoglu \& Tendick, 2000).

During the examination of an object, the haptic system acquires tactile information as well as information about arm displacement in conjunction with signals of applied force (i.e. kinesthesis information) (Clark \& Horch, 1986). Yet information regarding the displacement or deformation of an object may also be obtained from the visual system (Lederman \& Klatzky, 2009). However, in many surgeries procedures for example, the visual field is typically occluded (as in MaxilloFacial Surgeries), thus the surgeon has to rely solely on the information obtained from the haptic system.

Perceptual learning has been most extensively studied in the visual and auditory domains (Goldstone, 1998). A major mechanism of perceptual learning is for percepts to become increasingly differentiated from each other. By differentiation, stimuli that were once psychologically fused together become separated. Recent studies argued for multisensory facilitation of unisensory learning (Seitz, Kim \& Shams 2006; Shams \& Seitz, 2008; Lehmann \& Murray, 2005), thus suggesting that visual-haptic training may enhance haptic-only performance. Additional training manipulation, which is often argued for enhancing performance, is Knowledge of Results (KR) (Adams, 1987). Yet, as indicated by Salmoni, Schmidt \& Walter (1984), most of the KR experiments failed to separate the temporary effects of KR manipulations from permanent effects (learning). They showed that when KR was withdrawn, performance was deteriorated.

Although its importance, training of stiffness perception has not been addressed systematically. In continuation to our previous study that addressed the effects of order and sensory modality in a single session training (Korman et al., 2011), in the current study we investigated the course of learning in unimodal stiffness perception and how it is affected by different sensory conditions and information feedback during training. Specifically, we examined: first, whether practice repetitions alone improve stiffness perception and, second, how the addition of KR and/or visual information during training affect unisensory haptic performance and learning.

\section{Method}

This is an Open Access article distributed under the terms of the Creative Commons Attribution-Noncommercial License 3.0, which permits unrestricted use, distribution, and reproduction in any noncommercial medium, provided the original work is properly cited. 
Setup: The apparatus included a computer, monitor, 3D eyeglasses, mouse, and the PHANToM Desktop ${ }^{1}$ a pen-like stylus arm gripped and moved as in handwriting (see Fig. 1).

Task and experimental design: The experimental task was a Two Alternative Forced Choice (2AFC) discrimination task (Gescheider, 1997). On each trial two targets were presented on the screen as two red squared plates. Across all conditions, participants had to probe the targets with the stylus and determine which target is softer. Each discrimination pair was comprised of one standard stiffness value of constant $0.25 \mathrm{~N} / \mathrm{mm}$, and one comparison value out of the 11 possible. The values were chosen in a preliminary pilot study (not reported here), to ensure that discrimination is possible in this range, though not too easy, in order to address possible improvements.

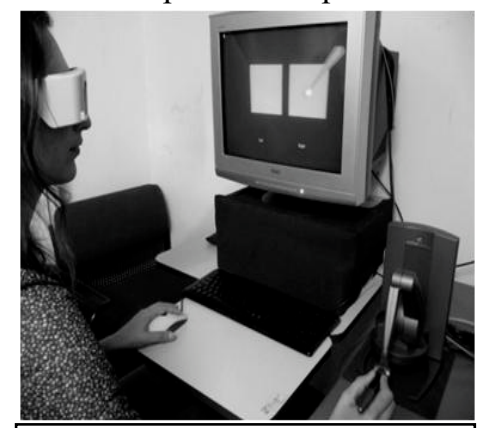

Two types of sensory stimuli were used in the study: Haptic (H) during target probing, only the haptic property of stiffness was presented (with fixed, non-matched

Figure 1. Experimental setup. The computer screen with two targets, Phantom, 3D glasses and a mouse.

visual component target square did not change its size and the stylus disappeared after contact with the target square to exclude visual feedback from stylus movement). Visual-Haptic (VH) - during target probing, matched (congruent in terms of visual target size and force-feedback) haptic and visual changes appeared for each of the targets under the application of force.

A total of 48 Technion students participated in the study. The experimental procedure included experiencing the discrimination task during two consecutive days. All subjects performed three blocks of the task in each day (test-training-test, see Fig. 2), separated by 5 min rest. Each block included 11 paired comparisons with the standard stiffness value; for each comparison, 10 repetitions were preformed, altogether 110 trials per block. Experimental session lasted approximately one hour in each day.

${ }^{1}$ Full technical descriptions of this virtual haptic system are available at http://www.reachin.se and http://www.sensable.com.

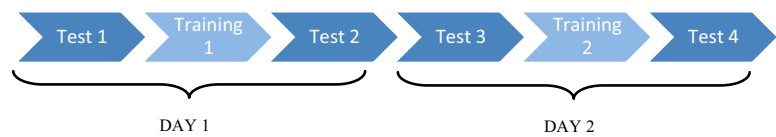

Figure 2. The time-course of training and re-tests

At training two variables were manipulated between participants: KR and/or addition of congruent visual information. Thus, two by two design was adopted: 4 groups, differing in training conditions were re-tested on haptic only condition performance before and after the training blocks on each training day (Table 1).

\begin{tabular}{|c|c|c|}
\hline & KR & NoKR \\
\hline H & H-KR (12 subjects) & H-nKR (12 subjects $)$ \\
\hline VH & VH-KR (12 subjects $)$ & VH-nKR (12 subjects $)$ \\
\hline
\end{tabular}

Table 1. Experimental groups

\section{Results}

Proportion of Correct answers (PC): We conducted repeated measures GLM analysis with $11 \times 6 \times 2 \times 2$ design (comparison's difficulty, block, KR, VH). Analysis of baseline performance in all experimental groups did not reveal significant differences. As expected, significant difficulty effects were found: Participants in all groups exhibited higher PC for easier comparisons (difficulty effect: $F(10,440)=315.11$, $\mathrm{p}<0.001)$. The results show significant and persistent improvement in unimodal stiffness discrimination ability induced by two-day practice, participants in all groups exhibited improvement from the first to the last block (practice effect: $F(5,220)=24.35, p<0.001$, see Fig. 3 for comparison of normalized gains attained by different groups).

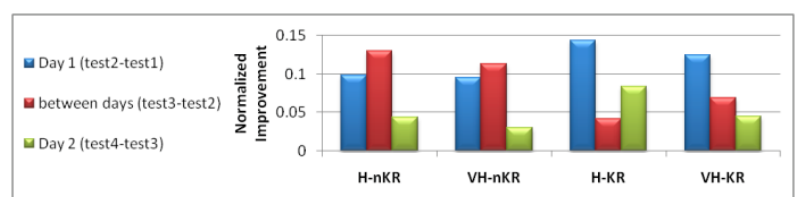

Figure 3. Normalized Improvements in PC at day 1 (blue), day 2 (green) and between the days (red)

Stiffness discrimination learning was evident in within and between session gains. Significant off-line, consolidation gains were found in all groups, as indicated by the red bars $(\mathrm{F}(1,44)=12.3 \mathrm{p}=0.001)$. However, these consolidation gains were smaller in the KR groups, possibly due to the relatively larger withinsession improvement observed during the first day in these groups (the blue bars). In addition, significant interaction between block, difficulty level and $\mathrm{VH}$ 
training was found (block*VH: $\mathrm{F}(5,220)=2.63$, $\mathrm{p}=0.024 ; \quad$ Block*VH*Difficulty: $\mathrm{F}(50,2200)=1.39$, $\mathrm{p}=0.038$ ), see Fig. 4. Addition of visual information resulted in higher PC scores during training blocks, but lower PC scores for haptic-only test blocks. These findings show that multisensory training does not necessarily facilitate later unimodal performance, as was suggested for the combination of visual and auditory modalities.

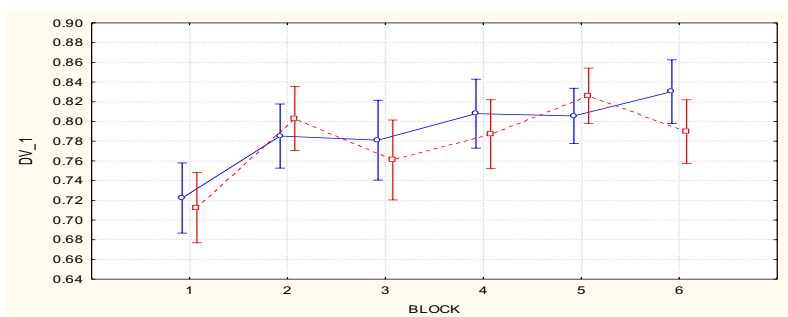

Figure 4. VH (red) vs $\mathrm{H}$ (blue) training in terms of absolute improvements in PC. Blocks 1, 3, 4 and 6 correspond to uni-haptic re-tests. Blocks 2 and 5 correspond to training sessions at dayl and day, respectively.

Decision Time (DT): We found temporary effects of $\mathrm{KR}$ and $\mathrm{VH}$ trainings on decision time: while $\mathrm{VH}$ training resulted in slower responses (Fig. 4), KR training induced faster responses, as compared with the control group (Fig.5). Nevertheless, as noted, these effects disappeared in the long run and were mainly evident during the training blocks (Significant interactions- Difficulty*Block: $\quad \mathrm{F}(50,2200)=1.59$, $\mathrm{p}=0.005 ; \quad$ Difficulty*Block*VH: $\mathrm{F}(50,2200)=1.43$, $\mathrm{p}=0.026) ; \quad$ Difficulty*Block*KR: $\mathrm{F}(50,2200)=1.7$, $\mathrm{p}=0.001$ ). In addition, DT analysis reveals negative consolidation effect $(\mathrm{F}(1,44)=15.8, \quad \mathrm{p}<0.001)$, it appears that off-line learning underlies the improvement in accuracy of discrimination between the last block on the first day to the first block on the second day, but that this improvement is accompanied by slower decisions.

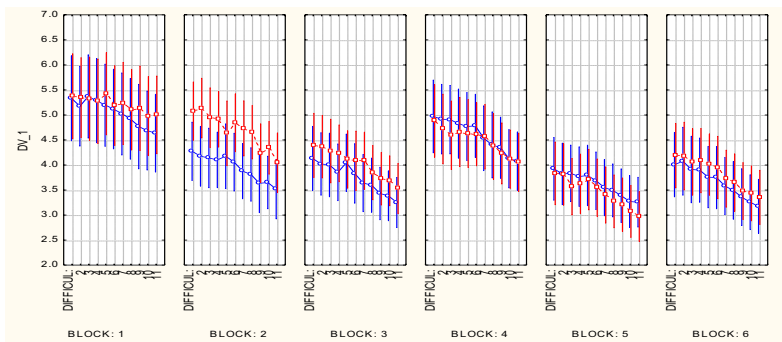

Figure 4. VH (red) vs. V (blue) training in terms of absolute improvements in Decision Time (sec). Block correspondence as in Fig. 3

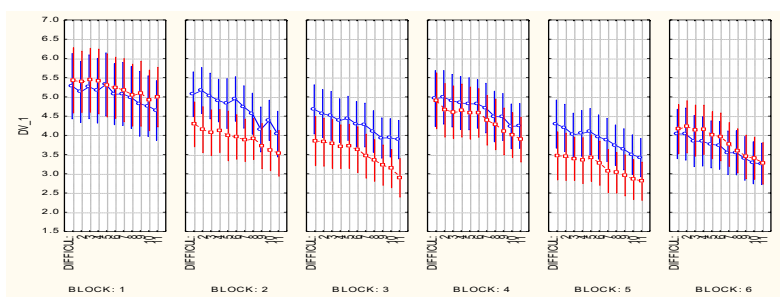

Figure 5. KR (red) vs NoKR (blue) training in terms of absolute improvements in Decision Time (sec). Block correspondence as in Fig. 3

\section{Conclusions}

Our study addressed the time course of learning a stiffness discrimination task. The results show that practice alone induces significant and persisting gains in stiffness discrimination. Mixed consolidation effects (off-line learning) were found: accuracy was improved, but slowing down of decisions time were observed between days of training. The addition of KR resulted in faster decisions but this improvement was transient. In contrast, the addition of visual information resulted in slower decisions, effect which disappeared in the long run. Importantly, visual-haptic training resulted in better stiffness discrimination during training, but this enhancement did not transfer to the haptic only posttraining re-tests. Haptic only training resulted in the best long-term discrimination gains. Altogether, we found that stiffness perception can be successfully trained and that multisensory training may have longterm effect on the uni-sensory stiffness discrimination ability. Information feedback has impact on the fast, within-session learning, but not on the consequences of the multi-session training. To our knowledge, this is the first study that specifically addressed the timecourse of stiffness discrimination learning and evaluated effects of training with additional modal or information feedback. Our findings have implications for optimization of training protocols in virtual environments for perceptual motor tasks relying on stiffness perception, such as surgery.

\section{Acknowledgments}

This work was supported by the SKILLS Integrated Project (IST-FP6 \#035005, http://www.skills-ip.eu) funded by the European Commission.

\section{References}

[1] Adams, J. A. (1987). Historical review and appraisal of research on the learning, retention, and transfer of human motor skills. Psychological Bulletin, 101, 41-74.

[2] Clark, F.J., Horch, K.W. (1986) Kinesthesia. In K. Boff, L. Kaufman, and J. Thomas (Eds.), 
Handbook of perception and human performance. New York: Wiley.

[3] Gescheider, G.A. (1997) Psychophysics: The fundamentals ( $3^{\text {rd }}$ edition). Mahwah NJ: Lawrence Erlbaum Associates. 3 p.

[4] Howell, J.N., Conatser, R.R., Williams, R.L., Burns, J.M., Eland, D.C. (2008) Palpatory diagnosis training on the virtual haptic back: performance improvement and user evaluations. J Am Osteopath Assoc 108(1): 29-36.

[5] Korman M, Weiss K, Cohen A, Reiner M, Gopher D. Effects of Practice and Sensory Modality on Stiffness Perception. Presence, 2011 Accepted

[6] Lederman, S.J., Klatzky, R.L. (2009). Haptic perception: A tutorial. Attention, Perception, \& Psychophysics 71 (7): 1439-1459

[7] Lehmann, S., \& Murray, M. M. (2005). The role of multisensory memories in unisensory object discrimination. Cognitive Brain Research, 24, 326-334.

[8] Salmoni AR, Schmidt RA, Walter CB (1984). Knowledge of results and motor learning: a review and critical appraisal. Psychol Bull 1984;5:355386.

[9] Seitz, A.R., Kim, R., Shams, L. (2006). Sound facilitates visual learning. Curr. Biol. 16, 14221427.

[10] Shams L, Seitz AR. (2008) Benefits of multisensory learning. Trends Cogn Sci. Nov;12(11):411-7. 